\title{
Does folic acid reduce congenital anomalies and adverse pregnancy outcomes in women received long-term low dose methotrexate?
}

\author{
Ahmed M. Farag*, Safaa A. Ibrahim \\ Department of Obstetrics and Gynecology, Faculty of Medicine Zagazig University, Zagazig, Egypt \\ Received: 15 November 2019 \\ Accepted: 07 December 2019 \\ *Correspondence: \\ Dr. Ahmed M. Farag, \\ E-mail: ahmed.farag@zu.edu.eg \\ Copyright: (c) the author(s), publisher and licensee Medip Academy. This is an open-access article distributed under \\ the terms of the Creative Commons Attribution Non-Commercial License, which permits unrestricted non-commercial \\ use, distribution, and reproduction in any medium, provided the original work is properly cited.
}

\begin{abstract}
Background: Methotrexate (MTX) which is folic acid antagonist is used to treat many diseases, including rheumatic, inflammatory, autoimmune disorders or malignancies; it is also used for the medical treatment of ectopic pregnancy and termination of pregnancy. The objective of this study was to assess the impact of folic acid in preventing congenital anomalies (CAs) in the fetus of pregnant that take long-term low dose methotrexate.

Methods: The study included 1569 with history of low dose methotrexate therapy <25 mg/week that stopped one month before pregnancy, they were divided into 3 groups; Group 1 comprised 272 pregnant women, Group 2 comprised 367 pregnant women both groups take folic acid 4-5 weeks preconception and for the first 12-13 weeks gestation, the dose in the $1^{\text {st }}$ group was $0.4 \mathrm{mg} /$ day while in $2^{\text {nd }}$ Group $4 \mathrm{mg} / \mathrm{day}$, Group 3 comprised of 930 pregnant disease-matched with no folic acid. Group 4 comprised of 1015 pregnant no underlying autoimmune and no folic acid intake.

Results: The overall rate of CAs and cardiovascular CAs were higher in fetuses of Group 3 (OR: 1.7; 95\% CI: 1.12.7) and (OR: $2.7 ; 95 \% \mathrm{CI}: 1.4-5.2)$ respectively compared to the overall rate of CAs and cardiovascular CAs in other groups. Other adverse outcomes as abortion, preterm delivery, preeclampsia, placenta abruption, PROM, low birth weight, admission to NICU, IUGR and IUFD were higher in Group 3 and 4 than in Group 1 and 2. There were no significant differences in umbilical arterial PH. The Apgar scores <7 at 1, $5 \mathrm{~min}$ and gestational age at delivery were higher in Group 1 and 2 than Group 3 and 4.

Conclusions: There was a certain reduction in methotrexate teratogenic effect on the fetuses with folic acid intake during pregnancy, these pregnancies might benefit from taking of folic acid in high doses before conception and during the first trimester of pregnancy and improvement in fetal and maternal outcomes but the explanation of this effect requires further study.
\end{abstract}

Keywords: Congenital abnormality, Fetal outcomes, Folic acid, Maternal, Methotrexate, Pregnancy

\section{INTRODUCTION}

Methotrexate (MTX) which is folic acid antagonist is used to treat many diseases, including rheumatic, inflammatory, autoimmune disorders or malignancies; it is also used for the medical treatment of ectopic pregnancy and termination of pregnancy. Dose varies according to indication of treatment, ranging from 2.5 $\mathrm{mg} /$ week to $30 \mathrm{mg} /$ week (for the treatment of rheumatoid arthritis), $1 \mathrm{gm} / \mathrm{m}^{2}$ of body surface area (for the treatment of some types of malignancy). MTX was found to be teratogenic in humans with high doses. ${ }^{1-4}$

Aminopterin (folate antagonist) and MTX has been associated with certain fetal anomalies: CNS anomalies, like anencephaly, spina bifida, hydrocephalus, and mental retardation; skeletal deformities, like absent or partial bones ossification, lambdoid sutures synostosis, 
micrognathia, cleft lip or palate, depressed nasal bone, hypertelorism, limb shortage, syndactyly, absent digits; and dextrocardia and IUGR. ${ }^{5,6}$

Folate deficiency may be due to insufficient dietary intake, defect in utilization, alcohol abuse, malabsorption, hepatic diseases, increased requirements in pregnancy and lactation, smoking, deficiency of enzymes responsible for FA formation or interference by drugs like anti-convulsant, methotrexate, oral contraceptives, sulfasalazine, trimethoprim and pyrimethamine. ${ }^{7-11}$

The functional folate insufficiency (one of the commonest nutritional deficiencies) is emerging in participating in maternal adverse outcomes as preeclampsia, which is a main cause of maternal mortality (15-20\% in developed countries) and morbidities (acute and long-term), perinatal deaths, PTL and IUGR. ${ }^{12,13}$ Despite the improvement of antenatal care, the scope of pre-eclampsia has not decreased. Several studies alluded that women who develop preeclampsia had increased peril of cardiovascular complications later on during life, in particular, hypertension, ischemic heart disease, venous thromboembolism, stroke, and even death. ${ }^{14-17}$ Primary prevention of these adverse pregnancy outcomes is a fateful project priority, including pre-conceptional approaches. ${ }^{18}$ Furthermore, finding inexpensive and simple ways to prevent these maternal and neonatal adverse outcomes is eminent scope. ${ }^{19-21}$

The target of this work to study the effect of folic acid supplement in higher dose (4 mg/day) before conception and during first trimester of pregnancy in comparison with the commonly recommended dose $(0.4 \mathrm{mg} /$ day $)$ on reducing the peril of all CAs and improving pregnancy outcomes in women received long-term low dose Methotrexate.

\section{METHODS}

This was a prospective observational study of women who were under low dose methotrexate $(<30 \mathrm{mg} / \mathrm{week})$ for a long time for treating autoimmune diseases and was planning for pregnancy, they were advised for stopping the drug for 1 month before pregnancy. Cases recruited from antenatal clinic (ANC) and/or maternity ward at Zagazig University Hospitals (ZUH), Zagazig, Egypt over the period from June 2015 to $28^{\text {th }}$ June 2018. The study sample included 639 pregnant women subjected to pre-conceptional MTX (367 take-ups $4 \mathrm{mg} /$ day, 272 takeups $0.4 \mathrm{mg} /$ day $4-5$-week pre-conception till 12-13-week gestation), 918 disease-matched comparison women without methotrexate, and 1015 comparison women without autoimmune diseases.

\section{Study protocol}

The study protocol was approved by ZUH ethical committee. Participating cases were counseled, the intervention was clearly explained, and an informed written consent was then taken from all of them. During booking (first visit), data recorded include age, parity, maternal weight and height, ethnicity, social condition (career of the mother and her partner), consanguinity and index of multiple deprivations. Medical history, obstetric history, and past history were taken. FA intake (onset and dose of supplementation), and time of first visit in pregnancy were collected plus additional information relating to pregnancy complications (gestational diabetes, antepartum hemorrhage, pregnancy-induced hypertension, and pre-eclampsia). Data of neonates included gestational age (from booking scans) and birth weight. GE Voluson E6, curvilinear probe 3.5 MHZ was used during the survey by a single sonographer.

At the second visit, compliance with vitamin intake was checked and the women had a urine pregnancy test, vitamin tablets were supplied for the next three months, and were inquired to get pregnant. If a woman did not conceive within the first three months, another threemonth supply of vitamin tablet was advised. If a woman did not conceive, she was excluded from the study.

The purpose of the third visit was confirmation of pregnancy by a urine pregnancy test and ultrasonography immediately after the first missed menstrual period. Compliance with vitamin intake was also checked and vitamin tablets were supplied.

At the fourth visit, compliance with vitamin intake was again evaluated and supplemented pregnant women who were 14 weeks' gestation (calculated from the first day of the LMP) were finally enrolled in the study and referred to a regional antenatal care clinic (ACC).

\section{Inclusion criteria}

- Pregnant women that take methotrexate not more than $30 \mathrm{mg} /$ day that stopped one month before the current pregnancy maternal age 20-35 years old, singleton fetus was eligible and approached for recruitment no smoking or consumption of alcohol.

\section{Exclusion criteria}

- Women with the following conditions were ineligible and excluded: maternal age $<20$ or $>35$ years old, multiple pregnancies, obesity $\left(\mathrm{BMI} \geq 25 \mathrm{Kg} / \mathrm{m}^{2}\right.$ ), diabetic or hypertensive patients.

Random assignment of women to receive $0.4 \mathrm{mg}$ of FA (referent cases) or $4 \mathrm{mg}$ of FA (treatment cases) daily. During ANC visit enrolment, general and abdominal examinations, past history and obstetric history were carried out, the fetal heart rate was checked, and findings were recorded. Routinely needed investigations, including $\mathrm{CBC}$ and urine analysis, liver and kidney function tests were arranged. In cases with prior regular menses, GA was calculated from the date of LMP. Otherwise, GA was estimated from early ultrasound scan 
(USS) at 7 weeks gestation and was verified by the $13^{\text {th }}$ week USS.

Ultrasound anomaly scan was accomplished at 20-24week gestation. If any anomaly was detected, women were counselled to continue the pregnancy or terminate it. Women were then followed up by the routine antenatal clinic protocol, re-examined every month until 28 weeks gestation and every 2 weeks until 36 weeks gestation then every week until delivery. Those who developed any adverse pregnancy outcome were seen more often or admitted accordingly to the maternity ward.

\section{Statistical analysis}

Was done using SAS version 8.02 (SAS Institute, Cary, NC, USA) for statistical analysis of data. First, the major maternal variables in the control and case groups were evaluated using a t-test for quantitative while $\mathrm{C} 2$ was used test for categoric variables in comparing cases with and without maternal autoimmune diseases as a reference.

The preponderance of other maternal diseases, drug supplementation particularly folic acid and multivitamins containing folic acid during pregnancy, in addition to pregnancy complications were compared between the control and case groups using odds ratios (OR) with $95 \%$ confidence intervals $(\mathrm{CI})$. We compared the appearance of specific $\mathrm{CA}$ in the case group and in all matched controls and adjusted OR with $95 \%$ CI were assessed in conditional logistic regression models. The distribution of specified CA-groups was evaluated in the children of pregnant women with a history of methotrexate therapy according to the dosage of folic acid supplement 4 $\mathrm{mg}$ /day versus $0.4 \mathrm{mg} /$ day from $4-5$ weeks before pregnancy till the end of the 1 st trimester during the survey of pregnancy outcomes.

Cases with CAs detected by ultrasound were divided into a single (affection of one organ only) and multiple (affecting two different organ systems at least and concurrent two or more CAs in the same case). The total prevalence of cases (birth + fetal) with CA diagnosed from the second trimester of pregnancy. Controls were the newborn infants without CA.

\section{Well-defined four CAs groups of informative offspring}

- Malformed fetuses diagnosed antenatally and terminated in the second, and very rarely in the third, trimester;

- Stillborn fetuses (late fetal death after the $28^{\text {th }}$ week of gestation); and

- Live-born infants. Each member of twins and triplets was evaluated separately. Isolated and multiple CAs were differentiated. Heart murmur without cardiological confirmation, deformations, minor anomalies, and genetic diseases were excluded.
Three-time windows were used for the detection of CAs in both cohorts

- Antenatally diagnosed fetal abnormalities in terminated pregnancies followed by a pathological description. There are four recommended ultrasound examinations during pregnancy and the number of ultrasound examinations ranged from 4 to12 in our study. All available medical records (ultrasound films, fetal pathological description, etc.) were collected to render a rigorous diagnosis of fetal defects.

- At birth, all deliveries of newborn infants and stillborn fetuses occurred in inpatient clinics of obstetrics and the attendants of labor were obstetricians during the period of the study. Most neonates were also examined by pediatricians in inpatient clinics of obstetrics. In addition, malformed infants were cared for in outpatient and inpatient pediatric clinics. An autopsy was compulsory in infant death, and it was requested in stillborn fetuses. If a completed pregnancy outcome certificate indicated any $\mathrm{CA}$, all available medical records (detailed physician's description, autopsy record, etc.) were obtained to evaluate clinical and pathological information as much as possible.

- At about the $12^{\text {th }}$ month after birth, all infants were called for personal examination and were checked by one pediatrician "blindly." If families did not participate in this examination, we repeated the invitation. If there was still no response, we contacted the pediatrician of the infant and obtained the case history, particularly data regarding CAs diagnosed after birth, a recent death, and serious and/or chronic disorders. Thus, all live-born infants except cases of postnatal death had a double check (birth and follow-up data regarding CA). Autopsy reports were available on all stillborn fetuses and infant demise.

\section{RESULTS}

Demographic data of pregnant women were similar in general (Table 1), but usage of systemic steroid or DMARD (drug modulating activity of rheumatoid arthritis) was lower in the women without underlying autoimmune disorder, also history of preterm birth or abortions were lower in the same group, this may due to underlying congenital anomalies in other groups. The proportion of diabetes mellitus, epilepsy, and CAs were 0.5 versus $0.2 \%, 0.5$ versus $0.1 \%$, and 2.2 versus $0.3 \%$, respectively, in the mothers of previous low doses of methotrexate therapy and other with no underlying autoimmune diseases.

The different fetal anomalies in cases with underlying autoimmune disorder and received folic acid (either 0.4 or $4 \mathrm{mg} /$ day) compared to those without folic acid supplement and pregnant women with no underlying autoimmune diseases were presented in Table 2, 3 . 
Table 1: Comparison between maternal characteristics in the studied groups.

\begin{tabular}{|c|c|c|c|c|c|c|c|c|c|c|}
\hline & \multicolumn{5}{|c|}{ Folic acid intake } & \multicolumn{5}{|c|}{ No folic acid } \\
\hline & $\begin{array}{l}0.4 \\
\text { mg/day } \\
(\mathrm{N}=272)\end{array}$ & $\begin{array}{l}4 \text { mg/day } \\
(N=367)\end{array}$ & OR & $(95 \% \mathrm{CI})$ & $\begin{array}{l}\text { p- } \\
\text { value }\end{array}$ & $\begin{array}{l}\text { Disease- } \\
\text { matched } \\
\text { group } \\
(\mathbf{N}=930)\end{array}$ & $\begin{array}{l}\text { Pregnant cases } \\
\text { with no underling } \\
\text { autoimmune } \\
\text { disease }(N=1015)\end{array}$ & OR & $(95 \% \mathrm{CI})$ & $\begin{array}{l}\text { p- } \\
\text { value }\end{array}$ \\
\hline $\begin{array}{l}\text { Maternal } \\
\text { age at } \\
\text { delivery (y) }\end{array}$ & $34.0 \pm 3.9$ & $33.4 \pm 4.6$ & & & 0.578 & $34.0 \pm 5.0$ & $33.3 \pm 4.3$ & & & 0.731 \\
\hline Primigravida & $\begin{array}{l}82 \\
(30.1 \%)\end{array}$ & $\begin{array}{l}112 \\
(30.5 \%)\end{array}$ & 1.06 & $\begin{array}{l}0.36- \\
3.14\end{array}$ & 0.866 & $\begin{array}{l}270 \\
(29.03 \%)\end{array}$ & $300(29.5 \%)$ & 1.22 & $\begin{array}{l}0.29- \\
5.17\end{array}$ & 0.917 \\
\hline Multipara & $\begin{array}{l}190 \\
(69.9 \%)\end{array}$ & $\begin{array}{l}235 \\
(69.5 \%)\end{array}$ & 0.64 & $\begin{array}{l}0.22- \\
1.85\end{array}$ & 0.569 & $\begin{array}{l}660 \\
(70.97 \%)\end{array}$ & $715(70.5 \%)$ & 0.54 & $\begin{array}{l}0.13- \\
2.22\end{array}$ & 0.595 \\
\hline $\begin{array}{l}\text { Previous } \\
\text { children } \\
\text { with } \\
\text { anomalies }\end{array}$ & $\begin{array}{l}32 \\
(11.7 \%)\end{array}$ & $\begin{array}{l}42 \\
(11.4 \%)\end{array}$ & 3.67 & $\begin{array}{l}1.06- \\
12.74\end{array}$ & 0.074 & $\begin{array}{l}108 \\
(11.6 \%)\end{array}$ & $10(0.9 \%)$ & 1.69 & $\begin{array}{l}0.18- \\
16.11\end{array}$ & $\begin{array}{l}< \\
0.001\end{array}$ \\
\hline \multicolumn{11}{|c|}{ Socio-economic class } \\
\hline High & $\begin{array}{l}99 \\
(36.4 \%)\end{array}$ & $\begin{array}{l}111 \\
(30.2 \%)\end{array}$ & \multirow{3}{*}{0.37} & \multirow{3}{*}{$\begin{array}{l}0.04- \\
3.25\end{array}$} & \multirow{3}{*}{0.619} & $\begin{array}{l}312 \\
(30.7 \%)\end{array}$ & $310(30.5 \%)$ & \multirow{3}{*}{1.69} & \multirow{3}{*}{$\begin{array}{l}(0.18- \\
16.11)\end{array}$} & \multirow{3}{*}{0.83} \\
\hline Middle & $89(32.7)$ & $\begin{array}{l}115 \\
(31.3 \%)\end{array}$ & & & & $\begin{array}{l}401 \\
(43.11 \%)\end{array}$ & $415(40.8 \%)$ & & & \\
\hline Low & 84 (30.9) & $\begin{array}{l}141 \\
(38.4 \%)\end{array}$ & & & & $\begin{array}{l}217 \\
(23.3 \%)\end{array}$ & $290(28.5 \%)$ & & & \\
\hline $\begin{array}{l}\text { Systemic } \\
\text { DMARD use }\end{array}$ & $\begin{array}{l}190 \\
(69.9 \%)\end{array}$ & $\begin{array}{l}235 \\
(69.5 \%)\end{array}$ & 0.64 & $\begin{array}{l}(0.22- \\
1.85)\end{array}$ & 0.569 & $\begin{array}{l}660 \\
(70.97 \%)\end{array}$ & $0(0 \%)$ & 0.54 & $\begin{array}{l}(0.13- \\
2.22)\end{array}$ & $\begin{array}{l}< \\
0.001\end{array}$ \\
\hline $\begin{array}{l}\text { Systemic } \\
\text { steroid use }\end{array}$ & $\begin{array}{l}82 \\
(30.1 \%)\end{array}$ & $\begin{array}{l}112 \\
(30.5 \%)\end{array}$ & 1.06 & $\begin{array}{l}(0.36- \\
3.14)\end{array}$ & 0.866 & $\begin{array}{l}270 \\
(29.03 \%)\end{array}$ & $15(1.4 \%)$ & 1.22 & $\begin{array}{l}(0.29- \\
5.17)\end{array}$ & $\begin{array}{l}< \\
0.001\end{array}$ \\
\hline $\begin{array}{l}\text { Prior preterm } \\
\text { delivery }\end{array}$ & $\begin{array}{l}32 \\
(11.7 \%)\end{array}$ & $\begin{array}{l}42 \\
(11.4 \%)\end{array}$ & 3.67 & $\begin{array}{l}(1.06- \\
12.74)\end{array}$ & 0.074 & $\begin{array}{l}108 \\
(11.6 \%)\end{array}$ & $10(0.9 \%)$ & 1.69 & $\begin{array}{l}(0.18- \\
16.11)\end{array}$ & $\begin{array}{l}< \\
0.001\end{array}$ \\
\hline $\begin{array}{l}\text { Prior } \\
\text { abortion }\end{array}$ & $\begin{array}{l}82 \\
(30.1 \%)\end{array}$ & $\begin{array}{l}112 \\
(30.5 \%)\end{array}$ & 1.06 & $\begin{array}{l}(0.36- \\
3.14)\end{array}$ & 0.866 & $\begin{array}{l}270 \\
(29.03 \%)\end{array}$ & $15(1.4 \%)$ & 1.22 & $\begin{array}{l}(0.29- \\
5.17)\end{array}$ & $\begin{array}{l}< \\
0.001\end{array}$ \\
\hline
\end{tabular}

Data are expressed as Mean \pm SD or number (\%). OR, odds ratio and CI, confidence interval

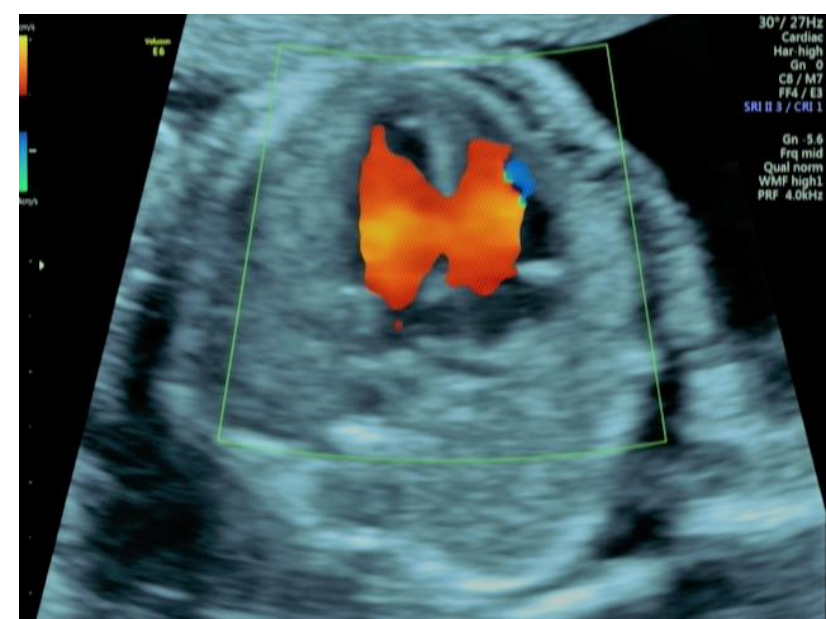

Figure 1: VSD. (4 chamber view with colour doppler image showing +ve flow across the sub valvular interventricular septal defect about $5 \mathbf{~ m m}$ ).

Pregnant women with underlying autoimmune disease without folic acid supplement had a higher peril of total rate of CAs in their progeny (OR: $1.7,95 \% \mathrm{CI}: 1.1-2.7$ ), excused principally by three isolated CA groups: isolated renal agenesis or dysgenesis, obstructive CA of the urinary tract (including 2 cases with cystic dysplasia), cardiovascular CA (including 12 cases with ventricular septal defect Figure 1 and 2, but the second most common CA was transposition of great vessels in 1 case) and by the multiple CAs group.

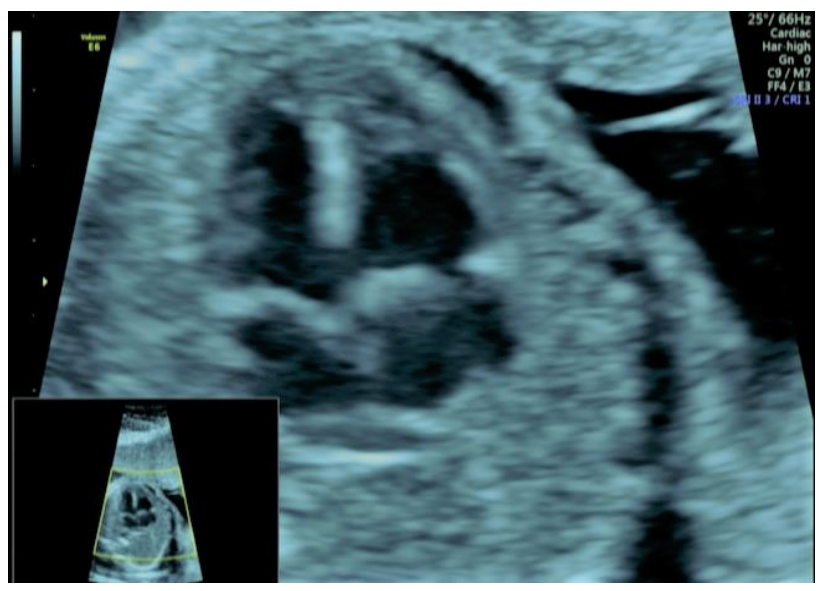

Figure 2: VSD (2D (greyscale) 4 chamber view showing +ve subvalvular VSD. 
Table 2: Estimation of different congenital abnormalities (CAs) in the studied groups with or without folic supplementation.

\begin{tabular}{|c|c|c|c|c|c|c|c|c|c|c|}
\hline & \multicolumn{5}{|c|}{ Folic acid supplementation $(\mathrm{N}=648)$} & \multicolumn{5}{|c|}{ No folic acid supplementation } \\
\hline & \multicolumn{2}{|c|}{$\begin{array}{l}\text { Group1 } \\
0.4 \text { mg/day }\end{array}$} & \multicolumn{2}{|c|}{$\begin{array}{l}\text { Group } 2 \\
4 \text { mg/day }\end{array}$} & \multirow[b]{2}{*}{$\begin{array}{l}\text { OR }(95 \% \\
\text { CI) }\end{array}$} & \multicolumn{2}{|c|}{$\begin{array}{l}\text { Group } 3 \\
\text { Disease matched- } \\
\text { control group No. } \\
(630)\end{array}$} & \multicolumn{2}{|c|}{$\begin{array}{l}\text { Group } 4 \\
\text { No past history of } \\
\text { methotrexate } \\
\text { therapy No }(815)\end{array}$} & \multirow[b]{2}{*}{$\begin{array}{l}\text { OR }(95 \% \\
\text { CI) }\end{array}$} \\
\hline & $\begin{array}{l}\text { No. } \\
(272)\end{array}$ & $\%$ & $\begin{array}{l}\text { No. } \\
\text { (367) }\end{array}$ & $\%$ & & No. (930) & $\%$ & $\begin{array}{l}\text { No. } \\
\text { (1015) }\end{array}$ & $\%$ & \\
\hline \multicolumn{11}{|l|}{ Isolated CAs } \\
\hline $\begin{array}{l}\text { Neural tube } \\
\text { defects }\end{array}$ & 0 & 0 & 0 & 0.00 & $-(-)$ & 2 & 0.21 & 0 & 0 & $2.3(0.7-7.4)$ \\
\hline $\begin{array}{l}\text { Cleft lip with or } \\
\text { without cleft } \\
\text { palate }\end{array}$ & 0 & 0 & 0 & 0.0 & 0 & 1 & 0.10 & 1 & 0.09 & $2.9(1.0-8.3)$ \\
\hline Cleft palate & 0 & 0 & 0 & 0.0 & 0 & 1 & 0.10 & 0 & 0 & $\begin{array}{l}3.3 \\
(0.8-13.6)\end{array}$ \\
\hline $\begin{array}{l}\text { Renal } \\
\text { a/dysgenesis }\end{array}$ & 1 & 0.36 & 0 & 0.00 & $-(-)$ & 2 & 0.21 & 0 & 0. & $\begin{array}{l}24.7 \\
(7.4-82.5)\end{array}$ \\
\hline $\begin{array}{l}\text { Obstructive CAs } \\
\text { of urinary tract }\end{array}$ & 2 & 0.72 & 1 & 0.27 & $\begin{array}{l}0.8(0.2- \\
3.0)\end{array}$ & 4 & 0.42 & 0 & 0 & $\begin{array}{l}8.5 \\
(2.6-28.1)\end{array}$ \\
\hline Hypospadias & 3 & 1.1 & 0 & 0.0 & $\begin{array}{l}1.0(0.4- \\
2.6)\end{array}$ & 3 & 0.31 & 1 & 0.09 & $0.7(0.2-2.7)$ \\
\hline $\begin{array}{l}\text { Undescended } \\
\text { testis }\end{array}$ & 2 & 0.72 & 0 & 0.0 & $\begin{array}{l}0.6(0.2- \\
2.7)\end{array}$ & 9 & 0.93 & 1 & 0.09 & $0.5(0.1-3.8)$ \\
\hline Ear CAs & 0 & 0 & 0 & 0.0 & $\begin{array}{l}3.9(0.9- \\
6.2)\end{array}$ & 8 & 0.84 & 0 & 0.00 & $-(-)$ \\
\hline $\begin{array}{l}\text { Cardiovascular } \\
\text { CAs }\end{array}$ & 1 & 0.36 & 0 & 0.0 & $\begin{array}{l}2.1(1.1- \\
3.5)\end{array}$ & 11 & 1.1 & 1 & 0.09 & $2.7(1.4-5.2)$ \\
\hline Clubfoot & 0 & 0 & 0 & 0.0 & $\begin{array}{l}0.8(0.2- \\
2.5)\end{array}$ & 13 & 1.3 & 1 & 0.09 & $0.8(0.2-3.5)$ \\
\hline Poly/syndactyly & 1 & 0.36 & 0 & 0.0 & $\begin{array}{l}0.4(0.1- \\
2.8)\end{array}$ & 2 & 0.21 & 0 & 0.0 & $1.2(0.3-5.1)$ \\
\hline $\begin{array}{l}\text { Other isolated } \\
\text { CAs }\end{array}$ & 1 & 0.36 & 0 & 0.0 & $\begin{array}{l}0.5(0.2- \\
1.7)\end{array}$ & 7 & 0.75 & 1 & 0.09 & $0.8(0.3-2.7)$ \\
\hline Multiple CAs & 1 & 0.36 & 0 & 0 & $\begin{array}{l}3.1(1.4- \\
6.9)\end{array}$ & 11 & 1.1 & 2 & 0.18 & $1.4(0.3-5.90$ \\
\hline Total & 12 & 6.2 & 1 & 0.27 & $\begin{array}{l}1.1(0.7- \\
1.7)\end{array}$ & 74 & 12.3 & 8 & 0.7 & $1.7(1.1-2.7)$ \\
\hline
\end{tabular}

However, the major intent of the study was to inspect whether folic acid supplement (either $0.4 \mathrm{mg}$ or 4 $\mathrm{mg} /$ day) 4-5 weeks preconception and for the first 12-13 weeks of pregnancy can reduce the peril of methotrexaterelated $\mathrm{CA}$; therefore, pregnant women with underlying autoimmune disease were differentiated into three subgroups: Group 1 who received $0.4 \mathrm{mg} /$ day, Group 2 who received $4 \mathrm{mg} / \mathrm{day}$ and Group 3 without folic acid supplement during the study pregnancy. Group 4, pregnant women with no underlying autoimmune diseases or folic acid supplement.

In fetus of pregnant women with folic acid supplementation $4 \mathrm{mg}$ /day pre-conceptional, only one case was affected by obstructive CAs of the urinary tract (Figure 3 and 4). There was no offspring with a neuraltube defect and cleft lip with or without cleft palate, cardiovascular or other anomalies. The total rate of CAs was lower in the fetus of pregnant women in Groups 1 and 2 (OR: 1.1, 95\% CI: 0.7-1.7) than in group 3 and 4. There was no offspring with a neural-tube defect or cleft lip \pm cleft palate in Group 1. One case of renal a/dysgenesis and 2 cases of obstructive CAs of the urinary tract and one case of cardiovascular CAs and multiple CAs in Group 2. The risk of cardiovascular CAs and multiple CAs has remained higher in Group 3 (11 cases for both). However, the total rate of CAs was significantly higher in the fetus of pregnant women without folic acid supplement (OR: 1.7, 95\% CI: 1.1-2.7), explained mainly by the higher rate of renal a/dysgenesis, urinary tract obstructive CAs, cleft lip \pm cleft palate, and cardiovascular CAs. Finally, it would be necessary to appraise the effect of folic acid according to the duration and dose of its supplementation and time of methotrexate therapy stoppage for its cumulative effect, three months before conception may be more effective, therefore, a beneficial preventative effect of folic acid can be more 
Table 3: Number (\%) of cases born to pregnant women with congenital anomalies according to the folic acid supplementation during the study pregnancy.

\begin{tabular}{|c|c|c|c|c|c|c|c|c|c|}
\hline & \multicolumn{4}{|c|}{ Folic acid } & \multicolumn{5}{|c|}{ No folic acid use } \\
\hline & \multicolumn{2}{|c|}{$\begin{array}{l}\text { Group1 } \\
0.4 \mathrm{mg} / \mathrm{day}\end{array}$} & \multicolumn{2}{|c|}{$\begin{array}{l}\text { Group } 2 \\
4 \text { mg/day }\end{array}$} & \multicolumn{2}{|c|}{$\begin{array}{l}\text { Disease- } \\
\text { matched } \\
\text { group }\end{array}$} & \multicolumn{3}{|c|}{$\begin{array}{l}\text { Cases without } \\
\text { underlying } \\
\text { autoimmune disease }\end{array}$} \\
\hline & $\begin{array}{l}\text { No. } \\
(272)\end{array}$ & $\%$ & $\begin{array}{l}\text { No. } \\
(367)\end{array}$ & $\%$ & $\begin{array}{l}\text { No. } \\
\text { (930) }\end{array}$ & $\%$ & No. (1015) & $\%$ & Total \\
\hline \multicolumn{10}{|l|}{ Isolated CAs } \\
\hline Neural tube defects & 0 & 0 & 0 & 0.00 & 2 & 100. & 0 & 0 & 2 \\
\hline $\begin{array}{l}\text { Cleft lip with or without cleft } \\
\text { palate }\end{array}$ & 0 & 0 & 0 & 0.0 & 1 & 0.50 & 1 & 0.50 & 2 \\
\hline Cleft palate & 0 & 0 & 0 & 0.0 & 1 & .100 & 0 & 0 & 1 \\
\hline Renal a/dysgenesis & 1 & 0.33 & 0 & 0.00 & 2 & 0.66 & 0 & 0. & 3 \\
\hline Obstructive CAs of urinary tract & 3 & 37.5 & 1 & 12.5 & 4 & 0.50 & 0 & 0 & 8 \\
\hline Hypospadias & 3 & 42.8 & 0 & 0.0 & 3 & 42.8 & 1 & 14.2 & 7 \\
\hline Undescended testis & 2 & 16.6 & 0 & 0.0 & 3 & 25.0 & 1 & 8.3 & 12 \\
\hline Ear CAs & 0 & 0 & 0 & 0.0 & 8 & 100 & 0 & 0.00 & 8 \\
\hline Cardiovascular CAs & 0 & 0.0 & 0 & 0.0 & 11 & 91.6 & 1 & 8.3 & 12 \\
\hline Clubfoot & 0 & 0 & 0 & 0.0 & 13 & 92.8 & 1 & 7.2 & 14 \\
\hline Poly/syndactyly & 1 & 33.3 & 0 & 0.0 & 2 & 66.7 & 0 & 0.0 & 3 \\
\hline Other isolated CAs & 1 & 9.09 & 0 & 0.0 & 8 & 72.7 & 2 & 18.1 & 11 \\
\hline Multiple CAs & 1 & 6.25 & 0 & 0 & 14 & 87.5 & 2 & 12.5 & 16 \\
\hline Total & 12 & 16.1 & 1 & 1.01 & 72 & 74.7 & 9 & 8.08 & 99 \\
\hline
\end{tabular}

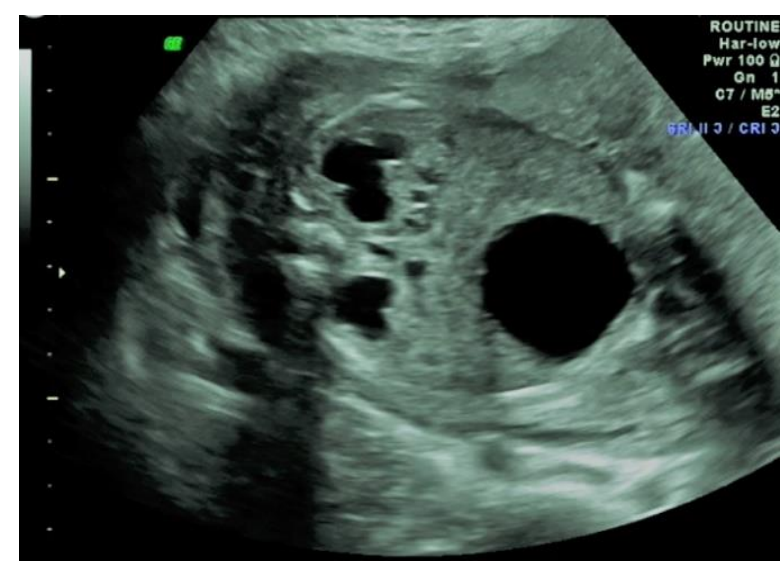

Figure 3: Obstructive urinary tract anomalies. (Grey scale coronal image of the abdomen showing bilateral mild to moderate hydronephrosis and marked distention of U.B.).

The incidences of preterm delivery were higher in the Group 3 and 4 than in the Group 1 and 2 (11.6\% versus 1.8\%); (OR: 6.8, 95\% CI: 1.2-3.2, $\mathrm{P}<0.005)$. Abortion was lower in Group 1 and 2 than others (OR: 2.9, 95\% CI: 1.05-8.83) against (OR: 4.39, 95\% CI: 2.5-6.5). The incidences of birth weight $<2500 \mathrm{gm}$ was higher in Group 3 and 4 than in Group 1 and 2 (OR: 4.8, 95\% CI:
1.8-2.9; $\mathrm{p}<0.05)$. There was no significant difference in umbilical arterial PH. The Apgar scores $<7$ at 1, 5 min was higher in Group 1 and 2 than Group 3 and 4 (OR: $4.39 ; 5.16)$ versus $(2.1 ; 2.9)$ respectively. The admission to NICU was higher in Group 3 and 4 than other groups (OR: 9.9, 95\% CI: 6.3-5.1) versus (OR: 1.5 , 95\% CI: $0.8-$ 2.93) also the gestational age at delivery was higher in Group 1 and 2; $\mathrm{p}<0.001$.

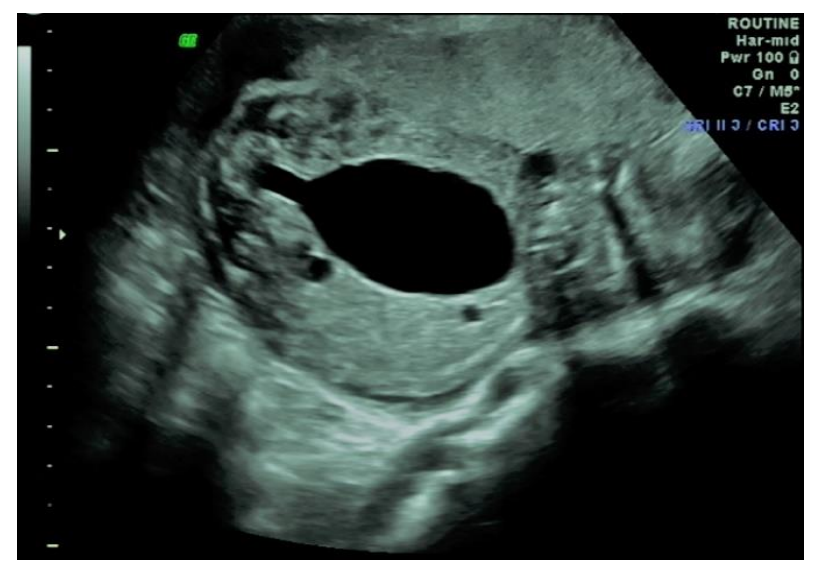

Figure 4: Obstructive urinary tract anomalies. (Grey scale coronal image of the pelvis showing characteristic keyhole deformity, typical for PUV (posterior urethral valve). 
Table 4: Number $(\%)$ of cases born to pregnant women with congenital anomalies according to the folic acid supplementation during the study pregnancy.

\begin{tabular}{|c|c|c|c|c|c|c|c|c|c|}
\hline \multirow[b]{3}{*}{ Study group } & \multicolumn{4}{|c|}{ Folic acid } & \multicolumn{5}{|c|}{ No folic acid use } \\
\hline & \multicolumn{2}{|c|}{$0.4 \mathrm{mg} / \mathrm{day}$} & \multicolumn{2}{|c|}{$4 \mathrm{mg} / \mathrm{day}$} & \multicolumn{2}{|c|}{$\begin{array}{l}\text { Disease-matched } \\
\text { group }\end{array}$} & \multicolumn{2}{|c|}{$\begin{array}{l}\text { Cases without } \\
\text { underlying } \\
\text { autoimmune disease }\end{array}$} & \multirow{2}{*}{$\begin{array}{l}\text { Total } \\
\text { No. }\end{array}$} \\
\hline & $\mathbf{N}$ & $\%$ & $\mathbf{N}$ & $\%$ & No. & $\%$ & No. & & \\
\hline \multicolumn{10}{|l|}{ Isolated CAs } \\
\hline Neural-tube defects & 0 & 0 & 0 & 0 & 2 & 100.0 & 0 & 0.0 & 2 \\
\hline $\begin{array}{l}\text { Cleft lip with or without cleft } \\
\text { palate }\end{array}$ & 0 & 0 & 0 & 0 & 1 & 50.0 & 1 & 50.0 & 2 \\
\hline Cleft palate & 0 & 0 & 0 & 0 & 1 & 100.0 & 0 & 0 & 1 \\
\hline Renal a/dysgenesis & 1 & 25 & 0 & 0 & 2 & 50.0 & 1 & 25.0 & 4 \\
\hline $\begin{array}{l}\text { Obstructive CAs of urinary } \\
\text { tract }\end{array}$ & 3 & 37.5 & 1 & $12.5 \%$ & 4 & 50.0 & 1 & 25.0 & 8 \\
\hline Hypospadias & 3 & 42.8 & 0 & 0 & 3 & 42.8 & 1 & 19.2 & 7 \\
\hline Undescended testis & 2 & 16.6 & 0 & 0 & 9 & 75 & 1 & 8.3 & 12 \\
\hline Ear CAs & 0 & 0 & 0 & 0 & 8 & 100 & 0 & 0 & 8 \\
\hline Cardiovascular CAs & 1 & 4.5 & 0 & 0 & 21 & 91 & 1 & 4.5 & 22 \\
\hline Club foot & 0 & 0 & 0 & 0 & 41 & 97.7 & 1 & 2.3 & 42 \\
\hline Poly/syndactyly & 1 & 28.6 & 0 & 0 & 2 & 71.4 & 0 & 0 & 7 \\
\hline Other isolated CAs & 3 & 31.25 & & $6.2 \%$ & 7 & 56.25 & 1 & 6.2 & 16 \\
\hline Multiple CAs & 3 & 30.07 & 0 & 0 & 11 & 64.28 & 2 & 7.6 & 26 \\
\hline Total & 17 & 14.5 & 1 & 1.2 & 74 & 78.4 & 8 & 5.6 & 100 \\
\hline
\end{tabular}

Table 5: Comparison between perinatal and maternal outcomes in the studied groups.

\begin{tabular}{|c|c|c|c|c|c|c|c|c|c|c|}
\hline & \multicolumn{5}{|l|}{ Folic acid } & \multicolumn{2}{|l|}{ No folic acid } & \multirow[b]{2}{*}{ OR } & \multirow[b]{2}{*}{$\begin{array}{l}(95 \% \\
\text { CI })\end{array}$} & \multirow[b]{2}{*}{$\begin{array}{l}p \text { - } \\
\text { value }\end{array}$} \\
\hline & $\begin{array}{l}0.4 \mathrm{mg} / \mathrm{day} \\
(\mathrm{N}=272)\end{array}$ & $\begin{array}{l}4 \\
\text { mg/day } \\
(\mathrm{N}=367)\end{array}$ & OR & $(95 \%$ CI $)$ & $\begin{array}{l}p \text { - } \\
\text { value }\end{array}$ & $\begin{array}{l}\text { Disease- } \\
\text { matched } \\
\text { group } \\
(\mathrm{N}=930)\end{array}$ & $\begin{array}{l}\text { With no } \\
\text { autoimmune } \\
\text { disease } \\
(\mathrm{N}=1015)\end{array}$ & & & \\
\hline $\begin{array}{l}\text { Admission to } \\
\text { NICU }\end{array}$ & $5(1.8 \%)$ & $6(1.6 \%)$ & 1.5 & $(0.80-2.93)$ & 0.183 & $212(22.7 \%)$ & $31(3.05 \%)$ & 9.9 & $(6.3-5.1)$ & $<0.05$ \\
\hline $\begin{array}{l}\text { Gestational age } \\
\text { at delivery } \\
\text { (weeks) }\end{array}$ & $\begin{array}{l}37.6 \\
(33.9-38.1)\end{array}$ & $\begin{array}{l}37.6 \\
(34.7-38\end{array}$ & & & 0.56 & $\begin{array}{l}36.7 \\
(25.6-37.7)\end{array}$ & $37.1(28.1 \%)$ & & & $<0.001$ \\
\hline PTL & $5(1.8 \%)$ & $0(0.0 \%)$ & 2.1 & $(2.12-21.84)$ & 0.002 & $108(11.6 \%)$ & $60(5.9 \%)$ & 6.6 & $(1.2-3.2)$ & $<0.005$ \\
\hline $\begin{array}{l}\text { Birth weight } \\
(\mathrm{g})\end{array}$ & $\begin{array}{l}2786 \\
(1958-3080)\end{array}$ & $\begin{array}{l}2863 \\
(1290- \\
3740)\end{array}$ & & & 0.235 & $\begin{array}{l}2364 \\
(610-3022)\end{array}$ & $\begin{array}{l}2778 \\
(1962-3432)\end{array}$ & & & 0.002 \\
\hline$<2500 \mathrm{~g}$ & $5(1.8 \%)$ & $1(0.2 \%)$ & 3.79 & $(1.30-11.04)$ & 0.025 & $112(12.04 \%)$ & $70(6.8 \%)$ & 4.8 & $(1.8 '-2.9)$ & $<0.05$ \\
\hline $\begin{array}{l}\text { Apgar Score } \\
1 \mathrm{~min}<7\end{array}$ & $4(1.4 \%)$ & $1(0.2 \%)$ & 4,39 & $(1.05-8.83)$ & 0.08 & $135(16.4 \%)$ & $70(6.8 \%)$ & 2.1 & $(2.5-6.5)$ & $<0.002$ \\
\hline $\begin{array}{l}\text { Apgar Score } \\
5 \mathrm{~min}<7\end{array}$ & $2(0.7 \%)$ & $0(0)$ & 5.16 & $(0.44-60.27)$ & 0.428 & $95(10.21 \%)$ & $13(1.2 \%)$ & 2.9 & $(1.2-2.8)$ & $<0.002$ \\
\hline $\begin{array}{l}\text { Umbilical } \\
\text { arterial pH }\end{array}$ & $\begin{array}{l}7.330 \\
(7.118-7.493)\end{array}$ & $\begin{array}{l}7.326 \\
(7.018-7.41\end{array}$ & & & 0.859 & $\begin{array}{l}7.318 \\
(7.228-7.366)\end{array}$ & $\begin{array}{l}7.316 \\
(7.057-7.425) \\
\end{array}$ & & & 0.815 \\
\hline Preeclampsia & $4(1.4 \%)$ & $1(0.2 \%)$ & 1.07 & $(0.38-2.96)$ & 0.892 & $55(5.9 \%)$ & $20(1.9 \%)$ & 5.1 & $\begin{array}{l}(2.5- \\
10.5)\end{array}$ & $<0.05$ \\
\hline $\begin{array}{l}\text { Placenta } \\
\text { abruption }\end{array}$ & $5(1.8 \%)$ & $1(0.2 \%)$ & 3.79 & $(1.30-11.04)$ & 0.025 & $112(12.04 \%)$ & $70(6.8 \%)$ & 4.8 & $\left(1.8^{\prime}-2.9\right)$ & $<0.05$ \\
\hline PROM & $4(1.4 \%)$ & $1(0.2 \%)$ & 2.9 & $(1.05-8.83)$ & 0.08 & $135(16.4 \%)$ & $70(6.8 \%)$ & 4.39 & $(2.5-6.5)$ & $<0.002$ \\
\hline IUGR & $4(1.4 \%)$ & $1(0.2 \%)$ & 1.07 & $(0.38-2.96)$ & 0.892 & $55(5.9 \%)$ & $20(1.9 \%)$ & 5.1 & $\begin{array}{l}(2.5- \\
10.5)\end{array}$ & $<0.05$ \\
\hline Abortion & $4(1.4 \%)$ & $1(0.2 \%)$ & 2.9 & $(1.05-8.83)$ & 0.08 & $135(16.4 \%)$ & $161(17.3 \%)$ & 17.31 & $(2.5-6.5)$ & $<0.002$ \\
\hline IUFD & $4(1.4 \%)$ & $1(0.2 \%)$ & 1.07 & $(0.38-2.96)$ & 0.892 & $55(5.9 \%)$ & $56(6.02 \%)$ & 5.1 & $\begin{array}{l}(2.5- \\
10.5)\end{array}$ & $<0.05$ \\
\hline
\end{tabular}

Data are expressed as number (\%) or median (range), OR (Odds Ratio), CI (Confidence Interval) and NA (Not applicable). 
Preeclampsia and placenta abruption were higher in Group 3 and 4 than Group 1 and 2 (OR: 5.1, 95\% CI: 2.510.5 ) versus (OR: $1.07,95 \% \mathrm{CI}: 0.38-2.96)$ and (OR: 4.8 , 95\% CI: $1.8-2.9$ ) versus (OR: $3.79,95 \%$ CI: $1.30-11.04)$. IUGR and IUFD were also higher in Group 3 and 4 (OR: 5.1, 95\% CI: $2.5-10.5$ ) versus (OR: $1.07,95 \%$ CI: 0.38 2.96). PROM was low in Group 1 and 2 than others (OR: 2.9, 95\% CI: 1.05-8.83) against (OR: 4.39, 95\% CI: 2.5$6.5)$.

PTL was lower in Group 2 than Group 1 (1.8\% versus $0 \%), \mathrm{p}=0.002$. Weight at birth $<2500 \mathrm{gm}$ was more in Group 1 than Group $2(1.8 \%$ versus $0.2 \%), p=0.025$. Unfortunately, the numbers were too low for convenient statistical analysis (Table 5).

\section{DISCUSSION}

Methotrexate teratogenic effect is known due to its antagonist effect to folic acid. The pre-conceptional low $(0.4 \mathrm{mg})$ doses of folic acid or folic acid $(0.8 \mathrm{mg})$ containing multivitamin, supplementation can reduce significantly the first appearance of neural-tube defects. ${ }^{22}$

In addition, as the Hungarian randomized controlled and cohort-controlled trials showed, pre-conceptional folic acid-containing multivitamin supplementation could reduce the occurrence of cardiovascular (especially construal) and urinary tract (especially obstructive) CAs. ${ }^{23,24}$ However, this primary prophylactic method was not able to decrease the multiple CAs occurrence. ${ }^{25}$ The rating of the population-based case-control dataset in Hungary showed that high doses of folic acid reduced the rate of isolated cardiovascular CAs and oro-facial clefts, but not the occurrence of multiple CAs..$^{23,26,27}$

Early population studies in the USA suggested that use of multivitamins with folate reduced the risk of nonsyndromic NTDs associated with other major birth defects. ${ }^{28-30}$ A major trial of the periconceptional use of folic acid in the prevention of NTDs by a randomized study conducted by the MRC was published in $1991 .^{31}$ From 1981 to 1996 there have been over 10 studies of periconceptional folate use and most of them reported lowered NTDs with folate use. ${ }^{32}$ Some regions of China have a high incidence of NTDs. ${ }^{33}$

The trial in China showed that periconceptional intake of $400 \mu \mathrm{g}$ of folic acid daily significantly decreased the peril of NTDs in areas of both high and low rates of such defects. ${ }^{34}$ Despite widespread education of the population about folic acid supplementation and food fortification, Kadir and colleagues did not find a decrease in the rate of NTDs in the general population in England and Wales in 1999.35

Shahraki et al, found that, administration of high dose folic acid may reduce the propagation of preeclampsia (PE), there is not sufficient data to favor that higher amount of folic acid administration can reduce the severity of presentation's signs or ameliorate the laboratory data and the onset of PE. ${ }^{36,37}$ Hernández-Díaz et al, suggested that intake of folic acid during pregnancy may decrease the risk of gestational hypertension. ${ }^{38}$

However, Li et al, suggested that $400 \mu \mathrm{g}$ folic acid daily intake only during early pregnancy cannot prevent the occurrence of preeclampsia or gestational hypertension. ${ }^{39}$

Earlier studies showed that supplement of folic acid was associated with decreased risk of preterm labor; Xiaohui et al, studied the effect of folic acid supplement more than 12 weeks on the peril of preterm birth and found that folic acid usage decreased the peril of preterm birth with a substantial dose-related response. ${ }^{40}$

Bukowski and colleagues reported that pre-conceptional supplement of folate is associated with up to $70 \%$ reduction in the incidence of spontaneous early preterm birth which proportionate inversely to the duration of folic acid usage. Pre-conceptional supplement of folate was related to spontaneous early preterm birth and not associated with other pregnancy complications. ${ }^{41}$ Wang et al, found that folate supplement decreased the risk of PTL while it was increased in those with abnormal BMI, although cases with higher BMI did not have this high risk when the adjusted analysis was executed. The protective effect of FA supplement for PTL was decreased in cases with $\mathrm{BMI} \geq 25 \mathrm{~kg} / \mathrm{m}^{2}{ }^{42}$

On the other hand, some studies did not find any benefit for folate supplement in the prevention of PTL; Saccone and Berghella, in their meta-analysis of randomized controlled trials on supplementation of folic acid in pregnancy to prevent PTL, found that folic acid supplementation during pregnancy does not prevent delivery $<37$ weeks. ${ }^{43}$ Martinussen et al, found that there was a lack of support for any benefit of folate intake on preterm birth or low birth weight. ${ }^{44}$

Nilsen et al, studied supplementation of folic acid and multivitamin and the risk of placental abruption and suggested that supplementation of folic acid and other vitamins during pregnancy may be associated with decreased risk of placental abruption. ${ }^{45,46}$

\section{CONCLUSION}

In conclusion, methotrexate has a cumulative effect on maternal tissue, so congenital anomalies may appear even after one month of its stoppage before pregnancy. low dose folate is not sufficient to prevent congenital anomalies in those women. High preconception dose (4 $\mathrm{mg}$ /day) at least 4-5 weeks and during the first trimester, can prevent anomalies in pregnant women. This can be explicated by the direct prophylactic effect of folic acid against CA, but this possible beneficial effect needs further studies to improve the medical care of those pregnant women. 


\section{ACKNOWLEDGMENTS}

Authors would like to thank all the colleagues who helped him to finish this work.

Funding: No funding sources

Conflict of interest: None declared

Ethical approval: The study was approved by the Institutional Ethics Committee

\section{REFERENCES}

1. Bawle EV, Conard, JV, Weiss L. Adult and two children with fetal methotrexate syndrome. Teratol. 1998;57:51-5.

2. Goffman D, Cole DS, Bobby P, Garry DJ. Failed methotrexate termination of pregnancy: a case report. J Perinatol. 2006;26:645-7.

3. Adam MP, Manning MA, Beck AE, Kwan A, Enns GM, Clericuzio C, Hoyme HE. Methotrexate/misoprostol embryopathy: report of four cases resulting from failed medical abortion. Am J Med Gen Part A. 2003;123(1):72-8.

4. Chapa JB, Hibbard JU, Weber EM, Abramowicz JS, Verp MS. Prenatal diagnosis of methotrexate embryopathy. Obstet Gynecol. 2003;101:1104-7.

5. Feldkamp M, Carey JC. Clinical teratology counseling and consultation case report: low dose methotrexate exposure in the early weeks of pregnancy. Teratol. 1993;47:533-9.

6. Lloyd ME, Carr M, McElhatton P, Hall GM, Hughes RA. The effects of methotrexate on pregnancy, fertility and lactation. QJM. 1999;92:551-63.

7. Charache S, Condit PT, Humphreys SR. Studies on the folic acid vitamins. IV. The persistence of amethopterin in mammalian tissues. Cancer. 1960;13:236-40.

8. McEvoy GK. AHFS drug information. Bethesda: American Society of Health-System Pharmacists. Methotrexate. 1996;751-9.

9. Hackmon R, Sakaguchi S, Koren G. Effect of methotrexate treatment of ectopic pregnancy on subsequent pregnancy. Can Fam Physician. 2011;57:37-9.

10. Vollset SE, Refsum H, Irgens LM, Emblem BM, Tverdal A, Gjessing HK, et al. Plasma total homocysteine, pregnancy complications, and adverse pregnancy outcomes: the Hordaland Homocysteine Study. Am J Clin Nutr. 2000;71:962-8.

11. Bergen NE, Jaddoe VW, Timmermans S, Hofman A, Lindemans J, Russcher H, et al. Homocysteine and folate concentrations in early pregnancy and the risk of adverse pregnancy outcomes: The generation $\mathrm{R}$ study. BJOG. 2012;119:739-51.

12. Sibai B, Dekker G, Kupferminc M. Pre-eclampsia. Lancet. 2005;365:785-99.

13. Steegers E, von Dadelszen P, Duvekot J, Pijnenborg R. Pre-eclampsia. Lancet. 2010;376:631-44.

14. Irgens HU, Reisaeter L, Irgens LM, Lie RT. Longterm mortality of mothers and fathers after pre- eclampsia: population-based cohort study. BMJ. 2001;323:1213-6.

15. Wilson BJ, Watson MS, Prescott GJ, Sunderland S, Campbell DM, Hannaford P, et al. Hypertensive diseases of pregnancy and risk of hypertension and stroke in later life: results from cohort study. BMJ. 2003;326:845

16. Haukkamaa L, Salminen M, Laivuori H, Leinonen $\mathrm{H}$, Hiilesmaa V, Kaaja R. Risk for subsequent coronary artery disease after preeclampsia. Am J Cardiol. 2004;93:805-8.

17. Bellamy L, Casas JP, Hingorani AD, Williams DJ. Pre-eclampsia and risk of cardiovascular disease and cancer in later life: systematic review and metaanalysis. BMJ. 2007;335:974-7.

18. Chang HH, Larson J, Blencowe H, Spong CY, Howson CP, Cairns-Smith S, et al. On behalf of the born too soon preterm prevention analysis group: preventing preterm births: analysis of trends and potential reductions with interventions in 39 countries with very high human development index. Lancet. 2013;381:223-34.

19. Yi Y, Lindemann M, Collins A, Snowball C. Economic burden of neural tube defects and impact of prevention with folic acid: a literature review. Eur J Pediatr. 2011;170:1391-400.

20. March of Dimes, The partnership of maternal, newborn and child health, save the children, World Health Organization: Born too soon: the global action report on preterm birth. Geneva: World Health Organization; 2012.

21. Bhutta ZA, Das JK, Rizvi A, Gaffey MF, Walker N, Horton S, et al. Lancet Interventions Review Group, Maternal and Child Nutrition Study Group: Evidence-based interventions for improvement of maternal and child nutrition: what can be done and at what cost? Lancet. 2013;382:452-77.

22. Czeizel AE, Dudas I. Prevention of the first occurrence of neural-tube defects by periconceptional vitamin supplementation. N Engl J Med. 1992;327:1832-5.

23. Czeizel AE. Reduction of urinary tract and cardiovascular defects by periconceptional multivitamin supplementation. Am J Med Genet. 1996;62:179-83.

24. Czeizel AE, Dobo M, Vargha P. Hungarian cohortcontrolled trial of periconceptional multivitamin supplementation shows reduction in certain congenital abnormalities. Birth Defects Res (PartA). 2004;70:853-61.

25. Czeizel AE, Medvecki E. No difference in the occurrence of multi malformed offspring after periconceptional multivitamin supplementation. Obstet Gynecol. 2003;102:1255-61.

26. Czeizel AE. Specified critical period of different congenital abnormalities: a new approach for human teratological studies. Congenit Anom. 2008;48:1039.

27. Czeizel AE, Puhó E, Bánhidy F. No association between periconceptional multivitamin 
supplementation and risk of multiple congenital abnormalities. A population-based case-control study. Am J Med Genet Part A. 2006;140A:2469-77.

28. Manning, SM, Jennings R, Madsen JR. Pathophysiology, prevention, and potential treatments of neural tube defects. Mental Retard Develop Disable Res Rev. 2000;6:6-14.

29. MRC vitamin study research group. Prevention of neural tube defects: results of the medical research council vitamin study. The Lancet. 1991;338(8760):131-7.

30. Khoury JM, Shaw GM, Moore CA, Lammer EJ, Mulinare J. Does periconceptional multivitamin use reduce the risk of neural tube defects associated with other birth defects? Data from two population-based case-control studies. Am J Med Gen. 1996;61:30-6.

31. Medical research council vitamin study research group. Prevention of neural tube defects: results of medical research council vitamin study. Lancet. 1991;338:131-7.

32. Lewis DP, Van Dyke DC, Stumbo PJ, Berg MJ. Drug and environmental factors associated with adverse pregnancy outcomes. Part I: Antiepileptic drugs, contraceptives, smoking, and folate. Ann Pharmacother. 1998;32:802-17.

33. Moore CA, Li S, Li Z, Hong SX, Gu HQ, Berry RJ, et al. Elevated rate of severe neural tube defects in a high-prevalence area in northern China. Am J Med Gen. 1997;73:113-8.

34. Berry RJ, Li Z, Erickson D, Li S, Moore CA, Wang $\mathrm{H}$, et al. Prevention of neural tube defects with folic acid in China. N Eng J Med. 1999;341:1485-90.

35. Kadir R, Sabin C, Whitlow B, Brockbank E, Economides D. Neural tube defects and periconceptional folic acid in England and Wales: retrospective study. Br Med J. 1999;319:92-3.

36. Wen SW, Guo Y, Rodger M, White RR, Yang Q, Smith GN, et al. Folic acid supplementation in pregnancy and the risk of pre-eclampsia-a cohort study. PLoS One. 2016;11(2):e0149818.

37. Shahraki AD, Dehkordi NZ, Lotfizadeh M. Comparison of high dose and low dose folic acid supplementation on prevalence, onset and severity of preeclampsia. Adv Biomed Res. 2016;5:192.

38. Hernández-Díaz S, Werler MM, Louik C, Mitchell AA. Risk of gestational hypertension in relation to folic acid supplementation during pregnancy. Am $\mathbf{J}$ Epidemiol. 2002;156(9and1):806-12.

39. Li Z, Ye R, Zhang L, Li H, Liu J, Ren A. Folic acid supplementation during early pregnancy and the risk of gestational hypertension and preeclampsia. Hyper. 2013;61:873-9.

40. Liu X, Lv L, Zhang H, Zhao N, Qiu J, He X, et al. Folic acid supplementation, dietary folate intake and risk of preterm birth in China. Eur J Nut. 2016;394015-0959-1.

41. Bukowski R, Malone FD, Porter FT, Nyberg DA, Comstock CH, Hankins GD, et al. Preconceptional folate supplementation and the risk of spontaneous preterm birth: a cohort study. Plos Med. 2009;6(5):1000061.

42. Wang Y, Cao Z, Peng Z, Xin X, Zhang Y, Yang Y, et al. Folic acid supplementation, preconception body mass index, and preterm delivery: findings from the preconception cohort data in a Chinese rural population. BMC Preg Childbirth. 2015;15:336.

43. Saccone G, Berghella V. Folic acid supplementation in pregnancy to prevent preterm birth: a systematic review and meta-analysis of randomized controlled trials. Eur J Obstet Gynecol Reprod Biol. 2016;1:42.

44. Martinussen KP, Bracken MB, Triche EW, Jacobsen GW, Risnes KR. Folic acid supplementation in early pregnancy and the risk of preeclampsia, small for gestational age offspring and preterm delivery. Eur J Obstet Gynecol Reprod Biol. 2015;195:94-9.

45. Nilsen RM, Vollset SE, Rasmussen SA, Ueland PM, Daltveit AK. Folic acid and multivitamin supplement use and risk of placental abruption: a populationbased registry study. Am J Epidemiol. 2008; 167:867-74.

46. Charles DH, Ness AR, Campbell D, Smith GD, Whitley E, Hall MH. Folic acid supplements in pregnancy and birth outcome: re-analysis of a large randomised controlled trial and update of Cochrane review. Paediatr Perinat Epidemiol. 2005;19:112-24.

Cite this article as: Farag AM, Ibrahim SA. Does folic acid reduce congenital anomalies and adverse pregnancy outcomes in women received long-term low dose methotrexate?. Int J Reprod Contracept Obstet Gynecol 2020;9:1-10. 